\title{
Integrando la Arteterapia como Herramienta Terapéutica en Trastornos del Comportamiento Alimentario: Estudio Piloto de un Programa de Intervención Grupal
}

\section{Integrating Art Therapy as a Therapeutic Tool in Eating Disorders: A Pilot Study of a Group Intervention Program}

Fecha de recepción: 12-07-2018

Fecha de aceptación: 18-07-2018
Carolina Martín

Facultad de Psicología. Universidad Autónoma de Madrid

Ana Gutiérrez Frutos

Facultad de Psicología. Universidad Autónoma de Madrid

Judith Pastor

Centro ITEM, Madrid Mª Paz López Centro ITEM, Madrid

Ana Rosa Sepúlveda

Facultad de Psicología. Universidad Autónoma de Madrid

\section{resumen/ahstract:}

Se describe un programa de intervención en arteterapia para pacientes con un trastorno del comportamiento alimentario, basado en el libro "Drawing from Within", de Lisa Hinz (2006). Este estudio piloto evaluó la aceptación del programa por parte de 13 pacientes y el impacto del programa. Fueron evaluados al inicio del estudio $\left(\mathrm{T}_{0}\right)$, al terminar la intervención $\left(T_{1}\right)$ y tres meses después $\left(T_{2}\right)$. Al finalizar el programa de intervención, se produjo una reducción en la sintomatología perteneciente a la bulimia y en la insatisfacción corporal de las pacientes. Hubo tendencias al cambio hacia una mejora en la autoestima y en la regulación emocional, sin embargo, no se obtuvo ningún cambio en la autoeficacia general. Estos cambios se mantuvieron en el tiempo. El programa fue altamente valorado por los pacientes, expresaron satisfacción con este tipo de intervención que utiliza la expresión artística para indagar sobre el mundo interior y las emociones de los pacientes. La intervención promueve mayor compromiso en las pacientes, si bien se necesita replicación de los resultados con muestras más amplias.

An Art Therapy intervention program for patients with an eating disorder is described based on the book "Drawing from within" by Lisa Hinz (2006). This pilot study evaluated the acceptance of the program by 13 patients and its impact. They were evaluated at the beginning of the study $\left(T_{0}\right)$, at the end of the intervention $\left(T_{1}\right)$ and three months after the intervention $\left(T_{2}\right)$. At the end of the intervention program, bulimia symptomatology and patient's body dissatisfaction were reduced. There was a changing tendency towards self-esteem and emotional regulation improvement. However, no change in general self-efficacy was achieved. These changes were maintained over time. The program was highly valued by patients, who were satisfied with this type of intervention, which uses artistic expression to inquire about the inner world and the emotions of the patients. The intervention promotes more commitment in patients, despite the results need to be replied with larger samples. 


\section{palabras clave/keywords:}

Trastornos alimentarios, arteterapia, intervención grupal, regulación emocional, autoestima, estudio piloto.

Eating disorder, art-therapy, group intervention, emotional regulation, self-esteem, pilot study.

\section{Introducción}

Los trastornos del comportamiento alimentario (TCA) son considerados trastornos psicosomáticos que comportan graves alteraciones en el comportamiento de la ingesta (Raich, 2011) y son uno de los problemas de salud más comunes que afectan a mujeres adolescentes y jóvenes en los países occidentales (Dalle, 2012). Con una etiología multifactorial, donde participan factores socio-culturales, el contexto interpersonal y familiar, los factores de vulnerabilidad personales (donde se incluyen factores genéticos y psicológicos) y los eventos vitales traumáticos (Striegel y Bulik, 2007). Estos trastornos se acompañan de múltiples consecuencias tanto médicas como psicológicas y sociales. Diversos autores señalan que la inanición y la reducción de la diversidad alimentaria provocan, entre muchas otras repercusiones físicas, bradicardia, embotamiento mental, problemas de sueño y alteraciones gastrointestinales severas, especialmente marcadas en presencia de conductas purgativas (Carvalho et al., 2013; Gerlinghoff y Backmund, 2004). En este sentido, Couturier, Kimber y Szatmari (2013) informan que la anorexia nerviosa es conocida por tener la tasa de mortalidad más alta que cualquier enfermedad mental, una tasa que aumenta 5,6\% por cada década en la persona que sigue presentando. Entre los aspectos psicológicos y sociales, destacan la rigidez mental, los trastornos del estado del ánimo, los problemas del control de los impulsos, el aislamiento (Billingsley-Marshall et al., 2013; Ruíz, Vázquez, Mancilla, López y Álvarez, 2010; Steiger, Labonté, Groleau, Turecki y Israel, 2013), que facilita una elevada emoción expresada por parte de los miembros familiares o circulo cercano, dificultando la relación y la comunicación familiar (Sepúlveda et al.,2009; Sepúlveda et al., 2014).

La evidencia empírica describe que las personas con TCA tienden a presentar problemas psicológicos coexistentes como una pobre autoestima e imagen corporal, perfeccionismo extremo, marcados problemas interpersonales y un estilo de afrontamiento poco adaptativo caracterizado por un mayor uso de estrategias de afrontamiento evitativas y un menor uso de estrategias centradas en el problema (Quiles y Terol, 2008). Estas dificultades mantienen la psicopatología del trastorno alimentario y obstruyen el cambio hacia la recuperación (Dalle, 2012).

Treasure, Smith y Crane (2011) describen que las personas que tienen un TCA, les suele resultar difícil manejar y regular las emociones. Puede que traten de evitar sentirlas, pensar en ellas y reconocerlas, y a veces actúan como si manifestar o hablar sobre sus emociones conflictivas tales como el sentirse herido, el enfado y la tristeza fueran inaceptables.

En este sentido, Cervera (2005), indica que además de normalizar la relación con la comida y los patrones alimentarios, es fundamental modificar las funciones psicológicas alteradas subyacentes con el objetivo de lograr una mejoría a largo plazo. 
En lo relativo a los antecedentes y al estado actual de la Arteterapia, la Asociación Americana de Arteterapia (2017) define la arteterapia como una forma útil de intervenir en salud mental en el que los pacientes, facilitado por el terapeuta, utilizan los medios de arte, el proceso creativo y la obra resultante para explorar sus sentimientos, resolver los conflictos emocionales, fomentar la conciencia sobre sí mismo, manejar el comportamiento y las adicciones, desarrollar habilidades sociales, mejorar la orientación de la realidad, reducir la ansiedad y aumentar la autoestima. El objetivo principal en la arteterapia es mejorar o restablecer el funcionamiento emocional de las personas y su sentido de bienestar personal.

Ganter et al. (2009) reconocen que la arteterapia en grupo es un método terapéutico muy extendido para los trastornos alimentarios, dado que las emociones que no se pueden experimentar en la terapia grupal cognitivo-conductual, a veces se pueden expresar mejor desde esta aproximación, con un proceso más creativo.

En este sentido, Potash, Chick, y Au Yeung (2013) han encontrado que tanto la visualización y como la creación del arte puede ser utilizados como una oportunidad de comunicación, punto de encuentro y de actividad reflexiva. Las psicoterapias con una aproximación artística son un lugar particularmente interesante en el tratamiento de las pacientes con trastornos de la alimentación, y por supuesto, en otras enfermedades donde las defensas personales son un obstáculo desde el inicio, la búsqueda de tratamiento, o para realizar cambios o en el proceso de recuperación (Dubois, 2010).

Hinz (2006) explica cómo funciona a nivel de procesamiento emocional la intervención terapéutica desde la arteterapia. El lado izquierdo del cerebro contiene el centro del lenguaje, aquí la información procesada es etiquetada, lineal y ordenada. El lado derecho del cerebro se orienta a procesar información visual-espacial. Esta información es intuitiva, emocional y espiritual. Sin palabras, y usando imágenes, la información se procesará más fácilmente en el lado derecho del cerebro y hay más probabilidad de que el contenido emocional dañado sea procesado e integrado. La capacidad de contener y explicar dos sentimientos opuestos hace que las imágenes del arte sean únicamente comunicativas y se pueda entender mejor esta ambivalencia que genera tanto malestar.

En la actualidad, la arteterapia ha demostrado ser una práctica efectiva (Schouten, Niet, Knipscheer, Kleber y Hutschemaekers, 2015) y se ha generalizado en una amplia variedad de entornos, incluyendo los hospitales, los centros psiquiátricos y de rehabilitación, los centros de bienestar, las escuelas y otros entornos clínicos y comunitarios (Asociación Americana de Arteterapia, 2017; Esteban y Garrido, 2013).

En relación a las distintas modalidades de psicoterapia, la Asociación Americana de Psicoterapia de Grupo (AGPA, 2014) define la psicoterapia de grupo como una forma especial de terapia en la que un pequeño número de personas se reúnen bajo la guía de un terapeuta capacitado profesionalmente para ayudarse a sí mismos y a los demás.

El arteterapia en grupo es un método terapéutico muy extendido en el tratamiento de los TCA (Ganter et al., 2009), ya que el grupo proporciona la oportunidad de contacto con personas que comparten sus sentimientos y experiencias personales, y que se enfrentan a los mismos obstáculos que ellos mismos (AGPA, 2014). 
Los investigadores como Burlingame et al. (2003) encontraron en un estudio de meta-análisis que utilizando la terapia grupal se logran mejorías y, en concreto, los pacientes que sufrían depresión y trastornos de la alimentación indicaron una mayor mejoría que aquellos con otros trastornos mentales.

Por otro lado, Dalle (2012) refiere que la terapia más eficaz en los TCA es la terapia cognitivo-conductual (TCC). Este es el tratamiento basado en la evidencia empírica para manejar a adultos con bulimia nerviosa, no obstante, su efectividad necesita ser mejorada, dado que el porcentaje de pacientes que logran una recuperación completa y duradera es entre el 55\% al $60 \%$.

Desde la intervención TCC, se trata de crear las condiciones para que la persona que tiene un trastorno de alimentación desarrolle las habilidades y la motivación para cambiar, llevando la alimentación reglada y dejando a un lado las conductas de seguridad (Treasure et al., 2011). En ocasiones, este objetivo con la terapia cognitivo-conductual no se logra cuando no se han abordado correctamente las emociones en varios aspectos de la vida de la persona, de modo que se estancan y no se ven cambios significativos, o mejoran pero teniendo recaídas constantes (Treasure et al., 2011). Es por ello, que en este estudio se plantea el uso combinado de la terapia cognitivo-conductual con un paquete de 10 sesiones grupales arteterapia.

El presente estudio cuenta con dos objetivos generales: 1) desarrollar un paquete de intervención desde la perspectiva de la arteterapia en pacientes con TCA, y 2) evaluar la viabilidad y aceptación de este programa piloto de intervención grupal.

Así como dos objetivos específicos: 1) disminuir los síntomas asociados a la conducta alimentaria, dificultades en la regulación emocional y problemas de imagen corporal de los pacientes participantes y 2) aumentar el nivel de autoeficacia y la autoestima tras la aplicación del programa.

Tras la intervención se espera que disminuyan los síntomas asociados a la conducta alimentaria, las dificultades en la regulación emocional y los problemas de imagen corporal, mientras se espera que aumente el nivel de autoeficacia y autoestima de los pacientes.

\section{Método}

\section{Diseño}

El programa conlleva 10 sesiones, con una duración total de tres meses, donde se realiza una evaluación pre-intervención $\left(\mathrm{T}_{0}\right)$, post-intervención $\left(\mathrm{T}_{1}\right)$ y una de seguimiento a los tres meses tras la intervención $\left(\mathrm{T}_{2}\right)$. Este estudio se basa en las distintas actividades y dinámicas de arteterapia específicas para la intervención en TCA, recogidas del libro "Drawing from Within" de Hinz (2006).

El programa recopila distintos ejercicios de arteterapia, que corresponde con distintos tópicos relevantes en el camino de la recuperación. En concreto, para tratar los siguientes temas relevantes en TCA: la conciencia de los efectos del trastorno alimentario, las influencias de la infancia en la enfermedad, la conciencia de sus propias necesidades, las habilidades de 
solución de problemas, las emociones y el efecto que estas tienen en sus vidas, la imagen corporal, la auto-aceptación a través de reconocer sus debilidades y fortalezas, la espiritualidad y la conexión con el interior.

\section{Participantes}

Los participantes son pacientes con TCA que reciben terapia en el centro privado ITEM en Madrid, España, y en la Fundación Fénix en Santo Domingo, República Dominicana. Del centro ITEM, hay 4 participantes y de la Fundación Fénix hay 9 participantes.

Los participantes son de ambos sexos, el 61,5\% corresponde al sexo femenino y el 38,5\% al sexo masculino. La edad media fue de 31 años $(D T=11,54)$. El 76,9\% estaban solteros, el 7,7\% vivía en pareja y el 15,4\% estaban casados. El 61,4\% de los participantes convivía con su familia.

En el momento de entrar en el estudio, el 30,8\% presentó un diagnóstico de anorexia nerviosa (AN), el $53,8 \%$ de bulimia nerviosa (BN) y el $15,4 \%$ de TCA no especificado (TCANE) según criterios del DSM-IV-R (APA, 2002). En el nivel de estudios, 38,5\% de los casos corresponden a bachillerato, $46,2 \%$ a estudios universitarios y el $15,4 \%$ estudios de Posgrado. El 53,8\% estaba trabajando frente al 46,2\% que estaba desempleado. El 38,5\% tenía pareja.

\section{Instrumentos}

Actitudes y síntomas alimentarios. Se evalúa con el instrumento EDI-II (Inventario de Trastornos de la Conducta Alimentaria; Garner, 1998). Este instrumento se utiliza para evaluar los síntomas de los trastornos alimentarios. Consta de 91 ítems que se responden en base a una escala tipo Likert, con 6 opciones de respuesta ("nunca" hasta "casi siempre o siempre"). El cuestionario ofrece puntuaciones en 11 distintas escalas clínicamente relevantes en los casos de trastornos de la conducta alimentaria, siendo estos: tendencia a la delgadez (TD), bulimia (B), insatisfacción corporal (BD), ineficacia (I), perfeccionismo (P), desconfianza interpersonal (ID), conciencia interoceptiva (IA), miedo a la maduración (MF), ascetismo (A), impulsividad (IR) e inseguridad social (SI). Puntuaciones mayores indican mayores actitudes y conductas de alimentación patológicas o rasgos psicológicos disfuncionales. Este cuestionario tiene una validez interna en su versión española muy aceptable, entre .84 y .92 para cada escala.

Satisfacción corporal. Se evalúa con el instrumento BSQ (Cuestionario de Imagen Corporal; Cooper et al., 1987; Raich et al., 1996). Este cuestionario mide la insatisfacción corporal, miedo a engordar, sentimientos de baja autoestima a causa de la apariencia y deseo de perder peso, y se obtiene una puntuación global. Consta de 34 ítems que pueden contestarse en una escala Likert con 6 opciones de respuesta: "nunca" hasta "siempre". A mayor puntuación, mayor insatisfacción corporal. La fiabilidad de la versión española es elevada de 93.

Regulación emocional. Se evalúa con el instrumento DERS (Escala de Dificultades en la Regulación Emocional; Gratz y Roemer, 2004; Hervás y Jódar, 2008). Esta escala evalúa cinco aspectos de la desregulación emocional a través de 28 ítems con una escala Likert de 5 puntos que va desde casi "nunca" hasta "casi siempre". Se obtiene una puntuación global 
y una puntuación para cada una de las cinco dimensiones evaluadas: atención-desatención, claridad-confusión, aceptación-rechazo, funcionamiento-interferencia y regulación-descontrol. Puntuaciones elevadas indican mayor desregulación emocional tanto en la escala global como en las demás escalas. Tiene una validez interna muy aceptable, entre .80 y .68 para cada escala.

Autoeficacia. Se evalúa con el instrumento EAG (Escala de Autoeficacia General; Baessler y Schwarzer, 1996; Martín-Aragón et al., 1997). La escala mide la percepción que tienen las personas de su capacidad para manejar acontecimientos o problemas. Los ítems están redactados en tiempo presente, de manera que el sujeto evaluado tiene que contestarlos pensando en el momento actual y en general cómo actúa ante determinadas circunstancias que se le plantean. Este instrumento consta de tres escalas: autoeficacia general, autoeficacia centrada en el manejo de situaciones difíciles e imprevisibles y autoeficacia centrada en el propio esfuerzo. Las respuestas son tipo Likert de 4 puntos con las siguientes opciones de respuesta que van desde "incorrecto" hasta "cierto". A mayor puntuación, mayor autoeficacia. La fiabilidad de la versión española es muy aceptable, de .84 para cada escala.

Autoestima. Se evalúa con el instrumento RSES (Escala de Autoestima de Rosenberg; Rosenberg, 1965; Echeburúa, 1995). Esta escala valora el grado de satisfacción de la persona consigo mismo, es decir, su autoestima. Consta de 10 ítems, 5 formulados en forma positiva y 5 en forma negativa, de modo que controla la tendencia a responder afirmativamente independientemente del contenido de la afirmación. Los ítems se responden en una escala tipo Likert de 4 puntos con respuestas que van desde "muy de acuerdo" hasta "muy en desacuerdo". Su fiabilidad en la adaptación española es aceptable de .84.

\section{Procedimiento}

Los participantes fueron 4 pacientes con TCA que recibían terapia en el centro privado ITEM en Madrid y 9 pacientes en la Fundación Fénix en Santo Domingo. Con la colaboración de los psicólogas/os respectivos, se seleccionaron qué pacientes con TCA cumplían los criterios de inclusión (presentar sintomatología TCA en curso, estar en etapa contemplativa o de acción) y se beneficiarían de esta intervención. Su participación era voluntaria y no tenía costes extras. Posteriormente, se firmó un consentimiento informado en el que se explicó de forma verbal y escrita todos los detalles referentes a la investigación y las normas del grupo, incluyendo la confidencialidad de los temas tratados y el respeto por la intimidad del resto de participantes. El primer grupo se llevó a cabo entre noviembre y diciembre del 2013, en el centro ITEM en Madrid. El segundo grupo de intervención, se llevó a cabo entre enero y febrero del 2014, en el centro Fundación Fénix en Santo Domingo.

Descripción del programa de intervención de Arteterapia. El desarrollo del paquete de intervención de Arteterapia constó de 10 sesiones de 90 minutos cada una, de 1 a 2 veces por semana. El programa de intervención se llevó a cabo en sesiones grupales y se usó como complemento a la terapia individual con enfoque cognitivo-conductual cada paciente recibía en individual semanalmente en cada centro.

Se desarrolló material de apoyo cuando fuera necesario en alguna sesión. Se incluyeron distintas actividades individuales, realizadas en el transcurso de la sesión grupal, y en algunas 
sesiones, ejercicios de arteterapia para casa, que se recogían al inicio de la próxima sesión. Cada sesión se organizó con relajación al inicio y al final, explicación de los ejercicios propuestos, tiempo para hacer el arte y tiempo para reflexionar sobre el dibujo/collage realizado. En cada sesión se propusieron de 1 a 3 ejercicios. Se completaron las evaluaciones antes y después de la intervención, y a los 3 meses de seguimiento. También completaron una escala de satisfacción de 11 ítems con una puntuación en el intervalo de 0-10.

En la tabla 1 se presentan los objetivos de cada una de las sesiones del programa.

Tabla 1. Objetivos de las sesiones del programa de intervención.

\begin{tabular}{|c|c|}
\hline Sesiones & Objetivos \\
\hline 1. Arte como terapia & $\begin{array}{l}\text { 1. Separar el trastorno de la persona. } \\
\text { 2. Reflexionar sobre cómo el origen de la enfermedad ha pasado de ser } \\
\text { una actividad útil a una actividad contraproducente. } \\
\text { 3. Practicar el utilizar el arte como forma de expresarse. }\end{array}$ \\
\hline $\begin{array}{l}\text { 2. Efectos del trastorno } \\
\text { alimentario }\end{array}$ & $\begin{array}{l}\text { 1. Analizar las similitudes y diferencias entre su yo interior y su yo } \\
\text { exterior. } \\
\text { 2. Explorar las expectativas familiares en cuanto al exterior e interior } \\
\text { de la persona. } \\
\text { 3. Trabajar en que lo que muestran al exterior es tan importante como } \\
\text { su interior. } \\
\text { 4. Enfatizar la importancia de los límites personales. } \\
\text { 5. Reconocer las debilidades, fortalezas y aprender de ambas. }\end{array}$ \\
\hline 3. Influencias de la infancia & $\begin{array}{l}\text { 1. Tener percepción de sus propias necesidades. } \\
\text { 2. Visualizar la diferencia entre las necesidades y los deseos. } \\
\text { 3. Separar las necesidades básicas que la persona perciba entrelazadas. }\end{array}$ \\
\hline $\begin{array}{l}\text { 4. Habilidades de solución de } \\
\text { problemas }\end{array}$ & $\begin{array}{l}\text { 1. Mirar honestamente a las personas en su vida que pudieran actuar } \\
\text { como barreras en su mejoría. } \\
\text { 2. Reforzar las conexiones con personas que apoyan la recuperación y } \\
\text { modifiquen, reduzcan y eliminen el resto de relaciones. } \\
\text { 3. Observar otras barreras, como la necesidad continua de ayudar a } \\
\text { otros dejándolos sin tiempo personal. } \\
\text { 4. Proporcionar la visión de mejoría que la paciente tiene de sí misma. } \\
\text { 5. Reenfocar a la paciente en qué tan lejos ha llegado, no en qué tanto } \\
\text { le falta. } \\
\text { 6. Comprender que los desafíos continuarán. }\end{array}$ \\
\hline $\begin{array}{l}\text { 5. Habilidades de solución de } \\
\text { problemas. }\end{array}$ & $\begin{array}{l}\text { 1. Reflexionar en cómo la imagen tiene la solución al problema } \\
\text { propuesto. } \\
\text { 2. Reenfocar que las soluciones al problema salieron de ellas mismas. } \\
\text { 3. Enfatizar la relevancia del proceso en las decisiones, antes que el } \\
\text { resultado en sí. } \\
\text { 4. Razonar que se aprende más de las derrotas que de las ganancias. } \\
\text { 5. Pensar minuciosamente en los problemas para llegar a decisiones } \\
\text { racionales. } \\
\text { 6. Reflexionar sobre la posibilidad que tienen de resolver sus problemas } \\
\text { sin esperar las decisiones de otros. } \\
\text { 7. Mejorar la confianza en su sabiduría interna. }\end{array}$ \\
\hline
\end{tabular}




\begin{tabular}{|c|c|}
\hline 6. Emociones & $\begin{array}{l}\text { 1. Diferenciar las cuatro emociones básicas } \\
\text { 2. Reconocer las emociones sencillamente como señales } \\
\text { de circunstancias significativas de la vida y que motivan el } \\
\text { comportamiento. } \\
\text { 3. Aprender que las emociones son temporales y que cambian cuando } \\
\text { cambia la situación que las produce. } \\
\text { 4. Analizar los estados de ánimo que se confunden. } \\
\text { 5. Determinar cómo esta confusión podría haber sido aprendida o } \\
\text { modelada. }\end{array}$ \\
\hline 7. Emociones & $\begin{array}{l}\text { 1. Observar la relación entre manifestaciones físicas de emociones y la } \\
\text { enfermedad o con otros problemas corporales. } \\
\text { 2. Enseñarles que los activadores emocionales tienen componentes } \\
\text { físicos, emocionales y cognitivos que pueden con práctica ser } \\
\text { identificados. } \\
\text { 3. Motivar a entender y expresar las emociones mejor para disminuir } \\
\text { condiciones físicas relacionadas con estrés. } \\
\text { 4. Volver a aprender la habilidad de escuchar y tener confianza de } \\
\text { nuevo en la sabiduría de sus cuerpos. } \\
\text { 5. Enseñar que las emociones no son siempre expresadas directamente } \\
\text { y que pueden ser expresadas a través de síntomas físicos. }\end{array}$ \\
\hline 8. Imagen corporal & $\begin{array}{l}\text { 1. Visualizar fortalezas físicas y otras habilidades que no conocen } \\
\text { conscientemente. } \\
\text { 2. Estudiar el conflicto de dependencia vs autonomía de las pacientes. } \\
\text { 3. Entender la separación e independización como un proceso natural } \\
\text { por el que todas las especies pasan. } \\
\text { 4. Concebir la independización como un proceso para encontrar las } \\
\text { preferencias y valores únicos de la persona. } \\
\text { 5. Confrontar sus distorsiones perceptuales. } \\
\text { 6. Comenzar a aceptar sus cuerpos como realmente son. }\end{array}$ \\
\hline 9. Autoaceptación & $\begin{array}{l}\text { 1. Demostrar y analizar cómo proyectan cualidades de sí mismas en el } \\
\text { arte. } \\
\text { 2. Analizar estas proyecciones, sacando a luz cualidades positivas. } \\
\text { 3. Demostrar que las cualidades de los pacientes admiran en otros son } \\
\text { cualidades que ellas mismas poseen y no se han dado cuenta. } \\
\text { 4. Reconocer, reclamar y admirar estas cualidades positivas de sí } \\
\text { mismas. } \\
\text { 5. Aceptar todos sus rasgos de personalidad. }\end{array}$ \\
\hline 10. Espiritualidad & $\begin{array}{l}\text { 1. Conectar con su espiritualidad para encaminarse a encontrar el } \\
\text { significado y propósito de sus vidas. } \\
\text { 2. Encontrar significado y placer en cosas que no se relacionen a su } \\
\text { enfermedad. }\end{array}$ \\
\hline
\end{tabular}

A continuación se presenta una descripción del programa de intervención:

Sesión 1. Expresión artística como terapia. En la primera sesión se les pidió que hicieran una imagen del trastorno alimentario. Al terminarlo, se discutió lo que habían creado y se trabajaron los objetivos propuestos. Como tarea para casa, se les pidió que hicieran un co- 
llage con una caja, de manera que el exterior de la caja reflejara lo que los demás veían de ellos y el interior lo que ellos veían de sí mismos.

Sesión 2. Efectos del trastorno alimentario. Cada uno de ellos reflexionó sobre qué se habían dado cuenta tras el ejercicio de la caja, sobre las diferencias y similitudes entre el interior y el exterior de la caja y cómo eran ellos mismos. Hicieron una lista de palabras que respondía a la caja collage y la explicaron. Posteriormente, se pidió que creasen una imagen abstracta que integrase el yo interior y el yo exterior de la caja collage. Los pacientes describieron los colores y formas que habían utilizado, y escribieron una lista de palabras referentes a la imagen creada y qué relación tenía con ellos mismos.

Sesión 3. Entender las influencias de la infancia. Comenzaron dibujando una relación familiar importante y sus necesidades dentro de ella, haciéndolo de manera metafórica, para sacar información más profunda. De manera que se reflexionó sobre los aspectos simbólicos del color, la línea y la forma, y se pasó a discutir sobre los objetivos del arte. Para explicar mejor el tema de las necesidades se sustentó con una imagen de la pirámide de necesidades humanas de Maslow para que aprendiesen qué son las necesidades, cuáles son los tipos de necesidades y cómo se deben satisfacer.

Sesión 4. Promocionar las habilidades de solución de problemas. Se planteó el camino de su recuperación, de las que son protagonistas, imaginando qué tipo de carretera y obstáculos se encontrarían en ese camino, y finalmente indicando en qué parte del camino se encontraban. Los pacientes realizaron una lista de palabras sobre este dibujo. Posteriormente se realizó otro ejercicio donde debían modificar el dibujo anterior para representar los cambios que posibiliten la recuperación y para resaltar el camino menos transitado que son aquellas tomas de decisiones que debían tomar solas sin ayuda de nadie más, esto lo hacían agregando, resaltando o eliminando áreas del camino según deseasen. Debían hacer una comparación del primer dibujo y de las modificaciones que le habían hecho.

Finalmente, se les dejó una tarea para casa que debían traer en la próxima sesión, donde se les pidió que pensasen y escribiesen un problema que les gustase resolver y lo dividan en tres o cinco elementos, asignándole un color a cada elemento. Luego, guardaron la lista y pegaron papeles de seda con los colores que representasen a cada elemento haciendo una imagen abstracta o real.

Sesión 5. Promocionar las habilidades de solución de problemas (cont.). Se comenzó con la discusión de la tarea, donde reflexionaron sobre esa imagen creada, visualizando cómo la imagen tenía la solución al problema propuesto. Luego, realizaron otro ejercicio que constó en pensar y escribir un problema o decisión que necesiten tomar, de modo que se pueda examinar los aspectos positivos y negativos del problema o decisión. Posteriormente, representaron a través de un collage los aspectos positivos en la mitad del folio, y los aspectos negativos en la otra mitad del folio. Contemplaron la imagen y hablaron sobre el proceso de construirla. Se debían llevar una tarea para casa que constó en realizar cuatro dibujos en un folio, que representasen las emociones básicas: enfado, tristeza, miedo y felicidad. En ella utilizaron elementos artísticos distintos para que se puedan diferenciar a simple vista unas de otras. 
Sesión 6. Recuperar las emociones. Se observó las imágenes que habían traído a la sesión y escribieron una lista de palabras de asociación sobre cada una de ellas. Se reflexionó sobre el proceso de discriminar las cuatro emociones básicas y sus imágenes resultantes. Se realizó un segundo ejercicio donde se les pidió que hagan 12 dibujos abstractos distintos que representen los siguientes estados de ánimo: enfado, ansiedad, curiosidad, depresión, emoción, miedo, culpabilidad, felicidad, indefensión, inocencia y tristeza. Con el nombre de cada estado de ánimo escrito detrás del dibujo, sin mirarlo y solo juzgándolo por las imágenes, debían clasificarlos en pilas como deseasen.

Sesión 7. Recuperar las emociones (cont.). Se les pidió que observasen la silueta de un cuerpo que se les ha dado, y se les explicó que representaba un mapa de sus cuerpos y cómo experimentan los sentimientos de enfado, tristeza, miedo y felicidad, más una emoción que agregasen en la línea en blanco. En la leyenda del mapa se representó cada sentimiento con un color distinto, y pintaron con cada color dónde sentían cada una de esas emociones en las distintas partes del cuerpo. Contemplaron el mapa de sensaciones corporales e hicieron una lista de palabras de asociación sobre él.

Posteriormente, se realizó un segundo ejercicio donde debían cerrar los ojos para buscar sensaciones positivas y negativas en el cuerpo. Centrándose en las sensaciones negativas, se realizó un dibujo de esa parte del cuerpo donde se sentía esa sensación y se utilizaron colores, tamaños y formas que definan esa molestia simbólicamente. Luego se le preguntó a esa parte del cuerpo: ¿Qué eres?, ¿Cómo te sientes? (centrado en sensaciones físicas y emociones), ¿Por qué te sientes así? y ¿Qué puedo hacer para ayudarte?

Sesión 8. Direccionar los problemas de imagen corporal. Debían utilizar plastilina para esculpir la imagen del animal con el que se habían identificado al imaginarse como animales. Escribieron una lista de palabras de asociación acerca de características físicas y personales del animal. Luego, se realizó un segundo ejercicio que comenzaba imaginándose ese animal que esculpieron en el ejercicio anterior como bebé. Se exploró cómo ha sido alimentado ese animal por sus padres, y cómo estos lo fomentan a que se haga independiente. A partir de ello se indagó cómo preferirían ser alimentados, y cómo alimentarían física y emocionalmente a su propio hijo, y cómo apoyarían su independencia.

Posteriormente, se realizó un tercer ejercicio donde se les pidió que dibujasen la silueta de sus cuerpos siendo lo más realista posibles. A partir de ello, se tumbaron encima del dibujo y el terapeuta trazó su silueta real del paciente. Comenzó la reflexión respecto a cómo se veían y cómo eran realmente en cuanto a su silueta corporal.

Sesión 9. Aumentar la autoaceptación. Debían hacer una lista de tres personas que no les agradan y debajo escribir cualidades de esas personas. Pensando en esas cualidades, debían hacer una máscara de cartulina. Hicieron una lista de palabras asociadas a la máscara que la describía física y psicológicamente. Con la lista de palabras escribieron un poema o párrafo describiendo la máscara y explicaron luego el significado. Más tarde realizaron un segundo ejercicio donde debían escribir el nombre de tres personas que admirasen y hacen la lista de cualidades de estas personas. Luego, realizaron una máscara teniendo estas cualidades en mente. Escribieron una lista de palabras que describía la máscara física y psicológicamente. 
Sesión 10. Fomentar la espiritualidad. Se explicó el concepto de laberinto como un viaje espiritual, un camino circular que se camina para la contemplación, meditación u oración. Se definieron las etapas del laberinto: entrada, centro y salida. De este modo, comenzaron a dibujar un laberinto que representase su viaje personal. Se da tiempo para la reflexión sobre la dificultad de mantener una conexión personal con ellas/o mismos.

\section{Análisis estadístico}

Los análisis fueron realizados con el programa SPSS versión 20.0. Se calcularon los descriptivos (medias, desviaciones típicas y/o porcentajes) de todas las medidas sociodemográficas, clínicas y psicológicas. Se utilizaron las medias para valorar los ítems de la escala de satisfacción de los participantes. Se utilizó la prueba de Shapiro-Wilk y se encontró que las variables no seguían una distribución normal. Las dos muestras de participantes se han unido tras verificar que no hay diferencias significativas en las variables independientes utilizando one-way ANOVA. Las variables independientes fueron comparadas a través del tiempo $\left(\mathrm{T}_{0}, \mathrm{~T}_{1}\right.$ y $\left.\mathrm{T}_{2}\right)$ con el test de Friedman. Cuando hay cambios significativos se realizan comparaciones por pares utilizando el test de Wilcoxon. Finalmente, se calculó la $d$ de Cohen para indicar la magnitud de la diferencia entre grupos según intervalos de tiempo. Según su autor (Cohen, 1988), un tamaño del efecto de $<0.4$ sería un efecto "pequeño", 0.4 sería un efecto "medio" y a partir de 0.75 sería un efecto "alto".

Aunque el número de análisis estadísticos es superior al permitido para fijar el error tipo I en el habitual 5\%, no se hicieron correcciones debido a la naturaleza exploratoria de la investigación. Por esa misma razón, a pesar de que se consideró como diferencia significativa el valor $p<0.05$, las tendencias a la significación $(p=0,05-0,20)$ también se tuvieron en cuenta.

\section{Resultados}

\section{Aceptabilidad y viabilidad del programa}

El 100\% de los pacientes completaron el programa, definido como asistir a un mínimo de 6 sesiones. La tasa de respuesta tras la intervención del programa $\left(\mathrm{T}_{1}\right)$ fue $100 \%(\mathrm{n}=13)$ y de un 76,9\% $(\mathrm{n}=10)$ en el seguimiento a los tres meses $\left(\mathrm{T}_{2}\right)$.

En la tabla 2 se muestra la puntuación media y desviación típica de los 11 ítems de la escala de satisfacción. Se contabilizaron las personas que superaban la puntuación media de 7 . En cuanto a los contenidos específicos del programa y su nivel de satisfacción, la mayoría consideró que les habían ayudado mucho los contenidos referentes a las necesidades básicas de las personas (puntuación $\geq 7: 69 \%$ de las pacientes; nota media global=7,08), la visión de su propia recuperación (puntuación $\geq 7: 92 \%$; nota media $=8,15$ ), las habilidades en la solución de problemas (puntuación $\geq 7: 77 \%$; nota media $=7,54$ ), y el reconocimiento y el manejo emocional (puntuación $\geq 7: 69 \%$; nota media $=7,62$ ). El programa también les ayudó a tener otra visión de su propia imagen corporal (puntuación $\geq 7: 100 \%$; nota media $=8,08$ ) y el conocerse y aceptarse a sí mismos (puntuación $\geq 7: 69 \%$; nota media= 7,31).

Se recogieron dos centros distintos, el Centro Ítem (Madrid) y la Fundación Fénix (Santo Domingo). Se realizaron los análisis estadísticos para examinar las diferencias entre ambas 
Tabla 2. Distribución de las medias de los ítems en la escala de satisfacción.

\section{Escala de Satisfacción}

\section{Contenido de los ítems}

$M(D T)$

1. ¿Cree que las sesiones han sido útiles para entender y trabajar mejor aspectos de su problema alimentario y elementos relacionados a ello?

2. En el programa se han proporcionado datos y consejos en cuanto a trastornos de la conducta alimentaria. ¿Le ha ayudado?

3. ¿Cree que el programa le ayuda a entender la relación del problema alimentario con las influencias de la infancia y las relaciones familiares?

4. ¿Podría decirnos si fue de ayuda conocer las necesidades básicas y cómo satisfacerlas?

5. ¿Las sesiones le han proporcionado una visión de su propia recuperación?

6. El programa trabaja en habilidades de solución de problemas, ¿ha aprendido algo de ellas?

7. ¿Las sesiones le han ayudado a reconocer y manejar las emociones?

8. ¿Ha sido útil conocer cómo ve su propia imagen corporal?

9. ¿Le han ayudado las sesiones a conocerse y aceptarse más?

10. En general ¿siente que las sesiones le han proporcionado herramientas que pueden ayudarles?

11. ¿Las sesiones eran lo que esperaba?

Nota: M = Media; DT = Desviación Típica.

muestras. La tabla 3 muestra que no existen diferencias estadísticamente significativas en cuanto a las 8 escalas principales del cuestionario EDI-II.

La tabla 4 muestra que tampoco existen diferencias estadísticamente significativas en cuanto a la desregulación emocional total (DERS), insatisfacción corporal (BSQ), autoeficacia general (EAG) y autoestima (RSE).

La tabla 5 muestra la comparación en el tiempo de los participantes (test de Friedman). Donde se muestra una mejora estadísticamente significativa en cuanto a la subescala de Bulimia (EDI-II), refiriéndose a la tendencia de tener pensamientos continuos sobre la comida y tener episodios de sobreingesta. La subescala de Conciencia Interoceptiva muestra una tendencia al cambio. En las comparaciones globales, no se han encontrado diferencias significativas en el resto de las subescalas del EDI-II. 
Tabla 3. Diferencias en las puntuaciones de inicio de los participantes en el EDI-II según muestra.

\begin{tabular}{lcccc}
\hline \multirow{2}{*}{ Variables (EDI-II) } & \multicolumn{4}{c}{$\boldsymbol{M}(\boldsymbol{D T})$} \\
\cline { 2 - 5 } & Centro ITEM & Fundación Fénix & $\boldsymbol{F}$ & $\boldsymbol{p}$ \\
\hline Obsesión por la delgadez & $11.50(3.42)$ & $12.56(5.73)$ & 0.12 & .74 \\
\hline Bulimia & $2.50(3.79)$ & $4.89(4.26)$ & 0.93 & .36 \\
\hline Insatisfacción corporal & $8.50(7.14)$ & $8.67(6.87)$ & 0.00 & .97 \\
\hline Ineficacia & $4.00(2.83)$ & $4.44(3.88)$ & 0.42 & .84 \\
\hline Perfeccionismo & $5.50(2.65)$ & $8.78(5.45)$ & 1.27 & .29 \\
\hline Desconfianza interpersonal & $3.25(1.71)$ & $1.78(2.86)$ & 0.89 & .37 \\
\hline Conciencia interoceptiva & $7.75(2.63)$ & $7.33(5.03)$ & 0.02 & .88 \\
\hline Miedo a la maduración & $1.25(1.26)$ & $4.56(3.32)$ & 3.58 & .09 \\
\hline
\end{tabular}

Nota $: \mathrm{M}=$ Media; DT = Desviación Típica; F = Estadístico F (ANOVA); $p=$ Nivel de significación.

Tabla 4. Diferencias en las puntuaciones al inicio entre las dos muestras en el DERS, BSQ, EAG y RSE.

\begin{tabular}{lcccc}
\hline \multirow{2}{*}{ Variables } & \multicolumn{4}{c}{$\boldsymbol{M}(\boldsymbol{D T})$} \\
\cline { 2 - 5 } & Centro ITEM & Fundación Fénix & $\boldsymbol{F}$ & $\boldsymbol{p}$ \\
\hline Desregulación emocional (DERS) & $58.75(6.40)$ & $75.56(22.66)$ & 2.03 & .18 \\
\hline Insatisfacción corporal (BSQ) & $110.75(46.01)$ & $110.67(24.56)$ & 0.00 & 1.00 \\
\hline Autoeficacia general (AEG) & $2.88(0.34)$ & $3.04(0.68)$ & 0.22 & .65 \\
\hline Autoestima (RSES) & $25.00(2.45)$ & $21.78(2.68)$ & 4.19 & .07 \\
\hline
\end{tabular}

Nota $: \mathrm{M}=$ Media; DT = Desviación Típica; $\mathrm{F}=$ Estadístico $\mathrm{F}($ ANOVA); $p=$ Nivel de significación.

Al hacer comparaciones por pares (prueba de Wilcoxon), se ha visto que la subescala de Bulimia muestra una mejora estadísticamente significativa entre el tiempo $\mathrm{T}_{0}-\mathrm{T}_{1}$, como en el $\mathrm{T}_{0}-\mathrm{T}_{2}$. En cuanto a la comparación $\mathrm{T}_{1}-\mathrm{T}_{2}$ de la subescala Bulimia se ha observado una 
tendencia al cambio. La subescala de Conciencia Interoceptiva muestra una mejora estadísticamente significativa entre el tiempo $\mathrm{T}_{0}-\mathrm{T}_{2}$, mientras muestra una tendencia al cambio en el tiempo $\mathrm{T}_{0-} \mathrm{T}_{1} \mathrm{y} \mathrm{T}_{1}-\mathrm{T}_{2}$.

Al ser un tamaño de muestra pequeño se ha analizado la $d$ de Cohen para examinar la magnitud de estos cambios, siendo los tamaños del efecto mayores cuando se evalúa la subes-

Tabla 5. Diferencias en función del momento en el cuestionario EDI-II.

\begin{tabular}{|c|c|c|c|c|c|c|c|c|}
\hline \multirow{3}{*}{$\begin{array}{c}\text { Variables } \\
\text { EDI-II }\end{array}$} & \multirow{3}{*}{$\mathbf{N}$} & $\begin{array}{l}\text { Línea } \\
\text { base }\end{array}$ & $\begin{array}{c}\text { Post- } \\
\text { inter- } \\
\text { ven- }\end{array}$ & $\begin{array}{l}\text { Segui- } \\
\text { miento }\end{array}$ & $\begin{array}{l}\text { Compara- } \\
\text { ciones }\end{array}$ & \multicolumn{3}{|c|}{ Comparaciones por pares } \\
\hline & & $N=13$ & $N=13$ & $N=10$ & $\mathrm{~T}_{0}-\mathrm{T}_{1}-\mathrm{T}_{2}$ & $\mathrm{~T}_{0}-\mathrm{T}_{1}$ & $\mathrm{~T}_{0}-\mathrm{T}_{2}$ & $\mathrm{~T}_{1}-\mathrm{T}_{2}$ \\
\hline & & $M(D T)$ & $\begin{array}{c}M \\
(D T)\end{array}$ & $M(D T)$ & $\begin{array}{c}\text { Estadístico } \\
\mathrm{F}(p)\end{array}$ & \multicolumn{3}{|c|}{ Prueba de Wilcoxon } \\
\hline $\begin{array}{l}\text { Obsesión } \\
\text { por la del- } \\
\text { gadez }\end{array}$ & 13 & $\begin{array}{l}12.23 \\
(5.00)\end{array}$ & $\begin{array}{l}10.08 \\
(5.36)\end{array}$ & $\begin{array}{c}9.90 \\
(5.57)\end{array}$ & $3.00(.22)$ & - & - & - \\
\hline Bulimia & 13 & $\begin{array}{c}4.15 \\
(4.12) \\
\end{array}$ & $\begin{array}{c}2.92 \\
(4.44) \\
\end{array}$ & $\begin{array}{c}1.70 \\
(3.09) \\
\end{array}$ & $9.17(.01)^{*}$ & $0.048^{\mathrm{a}}$ & $0.011^{\mathrm{b}}$ & $0.057^{\mathrm{a}}$ \\
\hline $\begin{array}{l}\text { Insatisfac- } \\
\text { ción cor- } \\
\text { poral }\end{array}$ & 13 & $\begin{array}{c}8.62 \\
(6.65)\end{array}$ & $\begin{array}{c}7.23 \\
(5.72)\end{array}$ & $\begin{array}{c}8.70 \\
(7.15)\end{array}$ & $2.32(.31)$ & - & - & - \\
\hline Ineficacia & 13 & $\begin{array}{c}4.31 \\
(3.47) \\
\end{array}$ & $\begin{array}{c}2.54 \\
(1.98) \\
\end{array}$ & $\begin{array}{c}3.30 \\
(2.31) \\
\end{array}$ & $2.24(3.33)$ & - & - & - \\
\hline $\begin{array}{l}\text { Perfecci- } \\
\text { onismo }\end{array}$ & 13 & $\begin{array}{c}7.77 \\
(4.90)\end{array}$ & $\begin{array}{c}6.92 \\
(4.59)\end{array}$ & $\begin{array}{l}4.50 \\
(2.37)\end{array}$ & $4.80(.91)$ & - & - & - \\
\hline $\begin{array}{l}\text { Desconfi- } \\
\text { anza inter- } \\
\text { personal }\end{array}$ & 13 & $\begin{array}{c}2.23 \\
(2.59)\end{array}$ & $\begin{array}{c}2.00 \\
(2.27)\end{array}$ & $\begin{array}{c}2.20 \\
(1.87)\end{array}$ & $0.89(.64)$ & - & - & - \\
\hline $\begin{array}{l}\text { Conciencia } \\
\text { interocep- } \\
\text { tiva }\end{array}$ & 13 & $\begin{array}{c}8.70 \\
(3.92)\end{array}$ & $\begin{array}{c}5.80 \\
(4.32)\end{array}$ & $\begin{array}{c}4.50 \\
(3.14)\end{array}$ & $5.06(.08)^{*}$ & $0.068^{\mathrm{b}}$ & $0.018^{\mathrm{c}}$ & $0.196^{\mathrm{a}}$ \\
\hline $\begin{array}{l}\text { Miedo a la } \\
\text { madura- } \\
\text { ción }\end{array}$ & 13 & $\begin{array}{l}3.80 \\
(3.19)\end{array}$ & $\begin{array}{c}3.70 \\
(2.36)\end{array}$ & $\begin{array}{l}3.90 \\
(2.33)\end{array}$ & $0.91(.63)$ & - & - & - \\
\hline
\end{tabular}

Nota $: \mathrm{N}=$ Frecuencia absoluta; $\mathrm{M}=$ Media; $\mathrm{DT}=$ Desviación Típica; $\mathrm{F}=$ Test de Friedman; $p=$ Nivel de significación. $*$ La diferencia es significativa al nivel $(\mathrm{p} \leq 0.05) ; * *$ La diferencia tiene tendencia a la significación $(\mathrm{p}=0.05-0.20)$. ${ }^{\mathrm{a}}$ Tamaño del efecto $(\mathrm{d}$ de Cohen $)<0.4$; ${ }^{\mathrm{b}}$ Tamaño del efecto ( $\mathrm{d}$ de Cohen $) \geq 0.4 ;{ }^{\mathrm{c}}$ Tamaño del efecto $(\mathrm{d}$ de Cohen $) \geq 0.75$. 
cala Bulimia en $\mathrm{T}_{0}-\mathrm{T}_{2}$. La subescala Bulimia obtiene un tamaño del efecto pequeño en $\mathrm{T}_{0} \mathrm{~T}_{1}$ y $T_{1}-T_{2}$. La subescala Conciencia Interoceptiva tiene un tamaño del efecto alto en $T_{0}-T_{2}$, un tamaño del efecto medio en $T_{0}-T_{1}$ y un tamaño del efecto pequeño en $T_{1}-T_{2}$.

En la tabla 6 se muestran las diferencias en las puntuaciones de las pruebas DERS, BSQ, EAG y RSE a través del tiempo. Se han encontrado cambios estadísticamente significativos en la Escala de Insatisfacción Corporal (BSQ). En las comparaciones globales no se han encontrado diferencias significativas en la Autoeficacia General (EAG). Sin embargo, se ha encontrado una tendencia hacia la significación en la Escala de Desregulación Emocional (DERS) y la Autoestima (RSE). Las comparaciones por pares de la Escala de Insatisfacción Corporal han mostrado una mejora significativa entre el tiempo $\mathrm{T}_{0}-\mathrm{T}_{2}$. Entre el tiempo de $\mathrm{T}_{0}-\mathrm{T}_{1}$ se ve una tendencia al cambio, al igual que entre el tiempo $\mathrm{T}_{1}-\mathrm{T}_{2}$.

$\mathrm{Al}$ analizar la magnitud de los cambios con la $d$ de Cohen, se puede observar un tamaño del efecto medio la Desregulación Emocional en $\mathrm{T}_{0}-\mathrm{T}_{2}$. La Insatisfacción Corporal tiene un tamaño del efecto pequeño en todas las comparaciones por pares. La Autoestima tiene un tamaño del efecto alto en $T_{1}-T_{2} y$ un tamaño del efecto medio en $T_{0}-T_{1}$.

Tabla 6. Diferencias en función del momento en cuestionarios DERS, BSQ, EAG y

\begin{tabular}{|c|c|c|c|c|c|c|c|c|}
\hline \multirow{3}{*}{ Variables } & \multirow{3}{*}{$\mathbf{N}$} & \multirow{2}{*}{$\begin{array}{c}\text { Línea de } \\
\text { base }\left(\mathbf{T}_{0}\right)\end{array}$} & \multirow{2}{*}{$\begin{array}{c}\begin{array}{c}\text { Post-inter- } \\
\text { vención }\left(\mathbf{T}_{\mathbf{1}}\right)\end{array} \\
N=13\end{array}$} & \multirow{2}{*}{$\begin{array}{c}\begin{array}{c}\text { Segui- } \\
\text { miento } \\
\left(\mathbf{T}_{2}\right)\end{array} \\
N=10\end{array}$} & \multirow{2}{*}{$\begin{array}{c}\begin{array}{c}\text { Compara- } \\
\text { ciones glo- } \\
\text { bales }\end{array} \\
\mathrm{T}_{0}-\mathrm{T}_{1}-\mathrm{T}_{2}\end{array}$} & \multicolumn{3}{|c|}{$\begin{array}{c}\text { Comparaciones por } \\
\text { pares }\end{array}$} \\
\hline & & & & & & $\mathrm{T}_{0}-\mathrm{T}_{1}$ & $\mathrm{~T}_{0}-\mathrm{T}_{2}$ & $\mathrm{~T}_{1}-\mathrm{T}_{2}$ \\
\hline & & $M(D T)$ & $M(D T)$ & $M(D T)$ & $\begin{array}{l}\text { Estadístico } \\
\mathrm{F}(p)\end{array}$ & \multicolumn{3}{|c|}{ Prueba de Wilcoxon } \\
\hline $\begin{array}{l}\text { Desregulación } \\
\text { emocional } \\
\text { (DERS) }\end{array}$ & 13 & $\begin{array}{c}70.38 \\
(20.44)\end{array}$ & $\begin{array}{c}64.85 \\
(23.33)\end{array}$ & $\begin{array}{c}62.00 \\
(17.65)\end{array}$ & $4,20(.12)^{* *}$ & 0.208 & $0.025^{\mathrm{b}}$ & 0.358 \\
\hline $\begin{array}{l}\text { Insatisfac- } \\
\text { ción corporal } \\
\text { (BSQ) }\end{array}$ & 13 & $\begin{array}{l}110.69 \\
(30.52)\end{array}$ & $\begin{array}{l}103.85 \\
(35.90)\end{array}$ & $\begin{array}{l}101.20 \\
(30.61)\end{array}$ & $7,80(.02)^{*}$ & $0.108^{\mathrm{a}}$ & $0.025^{\mathrm{a}}$ & $0.059^{\mathrm{a}}$ \\
\hline $\begin{array}{l}\text { Autoeficacia } \\
\text { general (AEG) }\end{array}$ & 13 & $2.99(0.59)$ & $3.22(0.57)$ & $\begin{array}{l}3.29 \\
(0.45)\end{array}$ & $0,80(.67)$ & - & - & - \\
\hline $\begin{array}{l}\text { Autoestima } \\
\text { (RSE) }\end{array}$ & 13 & $\begin{array}{l}22.77 \\
(2.95)\end{array}$ & $21.38(3.02)$ & $\begin{array}{l}24.10 \\
(3.21)\end{array}$ & $4.77(.09)^{* *}$ & $0.074^{\mathrm{b}}$ & 0.305 & $0.032^{\mathrm{c}}$ \\
\hline
\end{tabular}

Nota $: \mathrm{N}$ = Frecuencia absoluta; $\mathrm{M}=$ Media; DT = Desviación Típica; F = Test de Friedman; $p=$ Nivel de significación. * La diferencia es significativa al nivel $(\mathrm{p} \leq 0.05) ; * *$ La diferencia tiene tendencia a la significación $(\mathrm{p}=0.05-0.20)$

.${ }^{\mathrm{a}}$ Tamaño del efecto $(\mathrm{d}$ de Cohen $)<0.4 ;{ }^{\mathrm{b}}$ Tamaño del efecto (d de Cohen) $\geq 0.4$; ${ }^{\mathrm{c}}$ Tamaño del efecto $(\mathrm{d}$ de Cohen) $\geq 0.75$. 


\section{Discusión}

El objetivo de este estudio fue la aplicación de un paquete de intervención de arteterapia dirigido a pacientes con trastornos alimentarios basado en el libro "Drawing from Within" de Hinz (2006) y, a su vez, evaluar la viabilidad y aceptación a través de un estudio piloto.

La muestra fue recogida en dos poblaciones distintas y no hubo diferencias estadísticamente significativas entre ambas en las variables psicológicas evaluadas. La adherencia de la intervención fue alta ya que el $100 \%$ de los participantes completaron el programa, y la tasa de respuesta al seguimiento fue de 76,9\%. En cuanto a la aceptación de la intervención, el contenido y la forma de las sesiones fue valorada satisfactoriamente por los participantes de ambas muestras.

Un beneficio de realizar terapia de grupo en este estudio está relacionado con un beneficio mutuo por realizar una dinámica grupal, ya que normalmente siguen terapia semanal individual. En concordancia a lo descrito por la Asociación Americana de Psicoterapia de Grupo (2014), los grupos ayudan a encontrar apoyo y ganar fuerza al compartir sus sentimientos y experiencias personales, ayudando al participante a permitirse hablar más públicamente. Además, ha proporcionado la oportunidad de contacto con otras personas que también tienen TCA, uno de los beneficios descritos por Gerlinghoff y Backmund (2004). Según comentan los participantes, a través de compartir con otras personas con la misma enfermedad se han sentido con menor soledad, y más seguros.

La arteterapia también presenta ciertos beneficios en sí misma. El objetivo principal en la arteterapia es mejorar o restablecer el funcionamiento emocional del paciente (Asociación Americana de Arte Terapia, 2017). En este estudio se ha podido apreciar este beneficio dado que ha habido una tendencia al cambio en la desregulación emocional. Los participantes han expresado que el arte les ha ayudado a comunicarse cuando les cuesta hacerlo verbalmente, descrito por Hinz (2006), y son menos propensas a censurarse verbalmente. También, ha permitido una mayor expresión emocional, dado que las emociones que no se pueden experimentar en la terapia grupal cognitivo-conductual, a veces se pueden expresar mejor desde esta aproximación (Ganter et al., 2009).

Los resultados de este estudio piloto muestran beneficios de participar en este tipo de sesiones complementarias a su tratamiento cognitivo-conductual. Los análisis estadísticos permiten apreciar que el programa de intervención ha producido cambios estadísticamente significativos en la subescala Bulimia de la prueba EDI-II, mientras que ha habido una tendencia al cambio en la subescala Conciencia Interoceptiva de la misma prueba. También ha habido cambios estadísticamente significativos en la Insatisfacción Corporal (BSQ), y una tendencia al cambio en la Desregulación Emocional y la Autoestima (RSE). Lo importante es que estos cambios se mantienen en el tiempo. El hecho de que haya habido cambios y tendencias al cambio hace que estos resultados sean prometedores de que, con una muestra más grande, se podría obtener resultados mayores. Otro estudio que ha utilizado complemento a la terapia cognitivo-conductual es el estudio de Halmi (2005) que encontró que la terapia cognitivo-conductual más la terapia interpersonal en TCA producen una diferencia substancial y cambios de larga duración. 
El nivel de autoeficacia general (EAG) no ha habido cambios estadísticamente significativos probablemente porque las sesiones no den las habilidades de autoeficacia necesarias. También puede haber sucedido que se haya utilizado una escala para medir al autoeficacia que no sea la adecuada. Probablemente una escala de autoeficacia que no fuera patológica hubiera sido más adecuada para este estudio.

Este estudio cuenta con algunas limitaciones que son importantes señalar. En primer lugar, la muestra es muy reducida, por lo que sería contraproducente generalizar los resultados de la misma. Como bien se ha expresado anteriormente, es un estudio piloto exploratorio que con una pequeña muestra, promete resultados mejores. Sería interesante promover un estudio con una muestra mayor, al igual que estudios de eficacia con este tratamiento con el fin de valorar si este tipo de aproximaciones terapéuticas son o no complementarias a la terapia cognitivo-conductual y ofrecen una mejoría en la tasa de recuperación. Otra limitación del estudio es el tiempo del programa de intervención, el cual se podría extender a seis meses $\mathrm{y}$ un seguimiento de seis meses para valorar mejor su eficacia. Finalmente, el hecho de que no exista un grupo control de comparación, supone una limitación que no permite evaluar la eficacia del programa implementado.

\section{Referencias}

American Art Therapy Association (2017). About Art Therapy. Recuperado de: https://arttherapy.org/about-art-therapy/

American Group Psychotherapy Association (AGPA, 2014). What is Group Psycotherapy?. Recuperado de: https:// www.agpa.org/home/practice-resources/what-is-group-psychotherapy-

Billingsley-Marshall, R.L., Basso, M.R., Lund, B.C., Hernandez, E.R., Johnson, C. L., Drevets, W.C., McKee, P.A. y Yates, W.R. (2013). Executive function in eating disorders: the role of state anxiety. International Journal of Eating Disorders 46 (4), 316-321.

Burlingame, G., Fuhriman, A. y Mosier, J. (2003). The Differential Effectiveness of Group Psychotherapy: A Meta-Analytic Perspective. Group Dynamics: Theory, Research, and Practice 7 (1), 3-12.

Carvalho, S., Soares, M., Marques, M., Maia, B., Pereira, A., Nogueira, V., Valente, J. y Macedo, A. (2013). Disordered eating behaviors and sleep disturbances. Eating Behaviors 14 (3) 192-198.

Cervera, M. (2005). Riesgo y prevención de la anorexia y la bulimia. Madrid: Grupo Anaya.

Cohen, J. (1988). Statistical power analysis for the behavioral science. Hillsdale: Elrbaum.

Couturier, J., Kimber, M. y Szatmari, P. (2013). Efficacy of family-based treatment for adolescents with eating disorders: a systematic review and meta-analysis. International Journal of Eating Disorders 46. (1), 3-11.

Dalle, R. (2012). Intensive cognitive behavior therapy for eating disorders. New York: Nova Science Publishers.

Dubois, A. (2010). Art-thérapie et addictions, l'exemple des troubles du comportement alimentaire. Annales MédicoPsycologiques 168 (7), 538-541.

Echeburua, E. (1995). Evaluación y tratamiento de la fobia social. Barcelona: Martínez Roca.

Esteban, A., y Garrido, R. (2013). Arteterapia en el ámbito de la salud mental. Sevilla: ANASART.

Ganter, C., Enck, P., Zipfel, S., et. al. (2009). Art Therapy in Groups and Eating Disorders- a Literature Survey. Gruppenpsychotherapie und Gruppendynamik 45 (3), 20-31. 
Garner, D.M. (1998). EDI-2, Inventario de Trastornos de la Conducta Alimentaria. Madrid: TEA Ediciones.

Gerlinghoff, M. y Backmund, H. (2004). Anorexia y bulimia. Entender y superar los trastornos alimentarios. Barcelona: Anglofort.

Halmi, K. A. (2005). The multimodal treatment of eating disorders. World Psychiatry 4(2),69-73.

Hervás, G., y Jódar, R. (2008). Adaptación al castellano de la Escala de Dificultades en la Regulación Emocional. Clínica y Salud, 19(2), 139-156.

Hinz, L. (2006). Drawing from within. Using art to treat eating disorders. Londres: Jessica Kingsley Publishers.

Kohlenberg, R. J. y Tsai, M. (1994). Functional Analytic Psychotherapy: A behavioral approach to treatment and integration. Journal of Psychotherapy Integration 4 (1), 175-201.

Martín-Aragón, M., Pastor, M. A., López-Roig, S., Rodríguez-Marín, J., y Terol, M. C. (1997). Escala de autoeficacia general: Valoración preliminar. In Poster presented at VI Congreso de Psicología Social. San Sebastián.

Potash, J., Ho, R., Chick, J. y Au Yeung, F. (2013). Viewing and engaging in an art therapy exhibit by people living with mental illness: implications for empathy and social change. Public Health 127 (8), 735-744.

Quiles, Y. y Terol, M. C. (2008). Afrontamiento y trastornos de la conducta alimentaria: Un estudio de revisión. Revista Latinoamericana de Psicología, 40 (2), 259-28.

Raich, R. M., Mora, M., Soler, A., Ávila, C., Clos, I., y Zapater, L. (1996). Adaptación de un instrumento de evaluación de la insatisfacción corporal. Clínica y Salud 7, 51-66.

Raich, R. M. (2011). Anorexia, bulimia y otros trastornos alimentarios. Madrid: Anaya.

Ruíz, A. O., Vázquez, R., Mancilla, J. M., López, X. y Álvarez, G. L. (2010). Funcionamiento familiar en el riesgo y la protección de trastornos del comportamiento alimentario. Universitas Psychologica, 9 (2), 447-45.

Schouten, K. A., de Niet, G. J., Knipscheer, J. W., Kleber, R. J., y Hutschemaekers, G. J. (2015). The effectiveness of art therapy in the treatment of traumatized adults: a systematic review on art therapy and trauma. Trauma, Violence, \& Abuse, 16(2), 220-228.

Sepúlveda A. R., Todd G., Whitaker W., Grover M., Stahl D. y Treasure J. (2009). Expressed Emotion in relatives of patients with eating disorders following skills training program. International Journal of Eating Disorders, 43(7), 603-610.

Sepúlveda A. R., Anastasiadou D., Rodríguez L., Almendros C., Andrés P., Vaz F. y Graell M. (2014). Spanish validation of the Family Questionnaire (FQ) in families of patients with an eating disorder. Psicothema, 26/3), 321-327.

Steiger, H., Labonté, B., Groleau, P., Turecki, G. y Israel, M. (2013). Methylation of the glucocorticoid receptor gene promoter in bulimic women: associations with borderline personality disorder, suicidality, and exposure to childhood abuse. International Journal of Eating Disorders 46 (3), 246-255.

Striegel, R y Bullik, C (2007). Risk factors for eating disorders. American Psychologist, 62, (3), 181-198.

Treasure, J., Smith, G. y Crane, A. (2011). Los trastornos de la alimentación: Guía práctica para cuidar de un ser querido. Bilbao: Desclée de Brouwer. 